\title{
N9 $0-22921$
}

\section{STEREOSCOPIC DISTANCE PERCEPTION}

\author{
John M. Foley \\ Department of Psychology \\ University of California \\ Santa Barbara, California
}

\section{INTRODUCTION}

Most of this article is concerned with limited cue, open-loop tasks in which a human observer indicates distances or relations among distances. By open-loop tasks I mean tasks in which the observer gets no feedback as to the accuracy of responses. At the end of the article, I will consider what happens when cues are added and when the loop is closed, and what the implications of this research are for the effectiveness of visual displays.

Errors in visual distance tasks do not necessarily mean that the percept is in error. The error could arise in transformations that intervene between the percept and the response. I will argue, however, that the percept is in error. I will argue further that there exist post-perceptual transformations that may contribute to the error or be modified by feedback to correct for the error.

\section{METHODS}

First, I will describe some experiments on binocular distance perception. The stimuli were points of light viewed in dark surroundings. These were in or near the horizontal eye-level plane. The variables that I use are illustrated and defined in figure 1 . The angle subtended by straight lines from a stimulus point to the rotation centers of the eyes is the binocular parallax of that point. (It is sometimes called the convergence angle or stimulus to convergence.) The binocular parallax and the horizontal direction, $\theta_{\mathbf{i}}$, serve as coordinates that specify the positions of points in the plane. The binocular disparity of one point relative to another is defined as the binocular parallax of the first, minus the binocular parallax of the second. Note that binocular disparity is a signed quantity; a farther point has a negative disparity relative to a nearer one. The two open dots correspond to the perceived positions of $r$ and $i$. The binocular parallax of the perceived position of a point is called the effective binocular parallax of the point. The difference between two effective binocular parallaxes is an effective binocular disparity. These perceptual variables are defined in the same way as the corresponding physical variables except that perceived distance, $D^{\prime}$, is substituted for physical distance, $D$, in each equation. I assume that perceived horizontal direction equals physical horizontal direction. There is evidence that this is correct under the conditions of my experiments.

Some of the experiments I will describe were done with stimulus points at different distances. Others were done by simulating the distance dimension stereoscopically. If the stimulus to vergence is not grossly different than the stimulus to accommodation, the results are very similar. Some of the experiments employed a fixation point; others allowed the observers to move their eyes freely. When disparities are small, the results are again very similar. 


\section{RELATIVE DISTANCE TASKS}

I will describe performance on two classes of distance tasks. The first are called relative distance tasks; they are tasks in which an observer adjusts the position of light points by remote control until they satisfy some relative distance criterion (Foley, 1978, 1980). Examples of such criteria are shown in figure 2 . In each case the view is from above; the oval represents the observer's head and the dots represent stimulus lights. In the apparent fronto-parallel plane (AFPP) task, one point of light is fixed and the observer moves other lights so that they appear to lie in the vertical plane through the fixed light that is parallel to the vertical plane through the eyes or, in other words, a plane that is perpendicular to straight ahead. The apparent equidistant circle (AEDC) task is very similar, except that the lights are set so that they are perceived to lie on a circle with the observer at the center. In the apparent distance bisection (ADB) task, one point is fixed and the observer adjusts a second point so that the distance between the two points is perceived to equal the distance from the observer to the near point.

Typical performances in these tasks are illustrated in the second row for three distances of the fixed point. In each task there is one distance at which the physical configuration corresponds to the perceived configuration. This distance is generally within the range of 1-4 $\mathrm{m}$. At other distances, there are systematic errors in the settings. At far distances, variable points are set too far, and at near distances, they are set too near, relative to accurate performance. Although there are individual differences in the magnitude of the errors, errors of this kind are reliably found. (For many observers, one side of the configuration is set closer than the other (skewing). This can be accounted for by a very small difference in magnification in the two eyes. This is incorporated in a general theory of binocular distance perception (Foley, 1980), but it is not considered in this
article.)

I propose that these errors can be explained by the misperception of the egocentric distance to the fixation point, or, in the absence of a fixation point, to a reference point that depends on the configuration of points. To test this idea we must consider how the pattern of disparities produced by the observer compares with the pattern of disparities corresponding to the physical configuration specified by the instructions. By pattern of disparities I mean the function that relates binocular disparity to direction. The left side of figure 3 shows this function for physically fronto-parallel planes (PFPP) at different distances and the right side shows the same function for AFPP at different distances. If all the error in the AFPP settings is due to the misperception of the distance to the fixation point, then the function for an AFPP should be identical to the function for a PFPP, but generally this will be a PFPP at another distance. This is what the experiments show. For example, an AFPP at $1.2 \mathrm{~m}$ has less disparity than a PFPP at $1.2 \mathrm{~m}$, but corresponds to the same disparity pattern as a PFPP at $1.45 \mathrm{~m}$. Patterns of disparities obtained in the AEDC task also correspond closely with disparities produced by physically EDCs at other distances. Thus, the experimental settings can be accounted for by the hypothesis that the observer misperceives the egocentric distance to the configuration and produces the pattern of disparities appropriate to the
misperceived distance.

This hypothesis has several important implications. First, the fact that the pattern of disparities changes with the distance to the fixed point implies that there is an egocentric distance signal related to the vergence of the eyes, and this egocentric distance signal is not accurate. Second, effective binocular disparity equals binocular disparity. This is illustrated in figure 1 . In general, the distance to point $r$ will be misperceived. But if $r$ is misperceived, any other point $i$ will also 
be misperceived, so that the difference between the effective binocular parallaxes equals the difference between the binocular parallaxes. I call this the effective disparity invariance principle.

The data from relative distance tasks may be used to infer the perceived distance to the fixation point or to the reference point. The simplest way to conceptualize this is $t$, imagine a more complete set of functions on both sides of figure 3. Then, for each pattern on the right, we find the matching pattern on the left. The distance on the right is the physical distance that corresponds to the perceived distance on the left. This perceived distance is a concave downward function of physical distance, as is shown by the solid line on the left side of figure 4 . When both physical distance and perceived distance are transformed to parallaxes, their relation becomes linear, as is shown by the solid line on the right side of this figure. I call the curved function on the left the reference distance function and the linear function on the right the reference parallax function.

\section{EGOCENTRIC DISTANCE TASKS}

Next consider a different class of tasks-egocentric distance tasks. An egocentric distance task is one in which an observer indicates the distance from herself or himself to visual targets (Foley, 1977, 1985). Several different indicators have been used, but I have relied on two, verbal reports of perceived distance and pointing with an unseen hand. In the pointing experiments a horizontal board just beneath the targets prevents the observer from seeing his or her hand or arm. I will describe two simple experiments.

In the first experiment the stimulus is a single light point in dark surroundings. It is straight ahead. Pointed distances and reported distances from such experiments are shown in figure 4. The smooth curves shown have parameters that are close to the average values fitted to the data of five observers (Foley, 1977). On the left, indicated distance is plotted against physical distance, and on the right, the same values are plotted as binocular parallaxes. The functions on the left have the same form as the reference distance function; those on the right, the same form as the reference parallax functions.

But there is a complication: Verbal and manual indicators do not agree, and neither, in general, agrees with the function inferred from the relative distance tasks, which tends to lie between the verbal and manual functions. Since the indicators do not agree, both cannot correspond to perceived distance. I have defined perceived distance as the distance inferred from the relative distance tasks. When expressed as parallaxes, this value and the values indicated by pointing and verbal reports are all linearly related. This means that egocentric distance tasks can be used to test the implications of the theory. It is very important, however, to distinguish between perceived distance and indications of it. In figure 4 only the solid lines derived from the relative distance tasks correspond to perceived distance and reference parallax; the other lines describe indicated distance and indicated parallax.

When the eyes move freely, there is one point the perceived distance of which is given by the reference distance function. I call this point the reference point. Perceived distances of all other points are determined by their disparities relative to this point. There are several ways to determine the reference point. The most obvious is to measure the effective parallax of each point in the configuration and then determine how these are related to the reference parallax function. This analysis has been carried out only for the case of two-point configurations (Foley, 1985). Here the 
parallax of the reference point is a weighted average of the parallaxes of the points, with the farther point tending to receive the greater weight. Thus the reference point need not correspond to any
point of the configuration, although sometimes it may.

\section{DISCUSSION}

Figure 5 is a schematic diagram illustrating the process of binocular distance perception. The visual system generates both binocular parallax and binocular disparity signals in response to the optic array. The binocular parallax signals determine a single reference point and its corresponding value of effective binocular parallax. Here this is shown as an outflow from an eye movement control center. For each point $i$, the disparity of $i$ relative to the reference point is added to the effective reference parallax to give the effective parallax of the point. This value undergoes an indicator-specific linear transform to yield the indicated binocular parallax, which, in turn, determines the response.

When multiple cues are present, including perspective cues, distance perception is more accurate; however, the evidence indicates that there are systematic errors in distance perception under most cue conditions. There are several studies that have examined apparent distance bisection under such conditions. Although results have varied widely, no study has found consistently accurate bisection over a wide range of distances. The most common result is that the farther interval is set larger than the nearer one. There are also several studies that have obtained verbal reports of perceived distance under multiple cue conditions. The data are often fitted with a power function and the power is generally less than 1. An experiment limited to distances less than $70 \mathrm{~cm}$ yielded an accelerating verbal report function and a decelerating pointing response function (Foley, 1977). When the inverse output transforms derived from binocular experiments are applied to these data, both verbal and manual responses yield the same parallax function with a slope of about 0.8 . The conclusion is that distance perception is generally inaccurate, even in the
presence of multiple cues.

How can we perform accurately with respect to distance when distance perception is inaccurate? I can only answer this speculatively because the experiments needed to answer it scientifically have not been done. I hypothesize that we learn to behave accurately on the basis of feedback. This learning cannot be once and for all because the errors that it compensates for vary continuously with changing cue conditions. I hypothesize that the output transforms that I have proposed to explain open-loop performance are modified by feedback to compensate for perceptual
errors.

What implications does this have for the design of visual displays? I would expect that most visual displays evoke erroneous distance percepts. I expect this because even a three-dimensional scene with multiple cues evokes erroneous percepts, and most displays both eliminate cues and introduce cue conflicts, both of which are associated with increasing errors. In principle, it might be possible to create a display that would evoke accurate percepts, at least in some limited domain, but I doubt the wisdom of attempting this. The perceptual-motor system is designed to make rapid compensation for certain forms of error, especially those that can be described by linear transforms of the reference parallax function. Displays that produce errors of this form should suffice to direct behavior. But every time a display is used to direct behavior in the real three-dimensional space, 
performance with feedback is necessary to calibrate the output transforms, just as performance with feedback is necessary when a three-dimensional scene directs behavior.

\section{REFERENCES}

Foley, J. M.: Effect of Distance Information and Range on Two Indices of Visually Perceived Distance. Perception, vol. 6, 1977, pp. 449-460.

Foley, J. M.: Primary Distance Perception. In Handbook of Sensory Physiology, R. Held, H. Leibowitz, and L. Teuber, eds., Berlin: Springer-Verlag, vol. 8, pp. 181-213.

Foley, J. M.: Binocular Distance Perception. Psych. Rev., vol. 87, 1980, pp. 411-434.

Foley, J. M.: Binocular Distance Perception: Egocentric Distance Tasks. J. Exp. Psych.: Human Percept. Perform., vol. 11, 1985, pp. 133-149. 


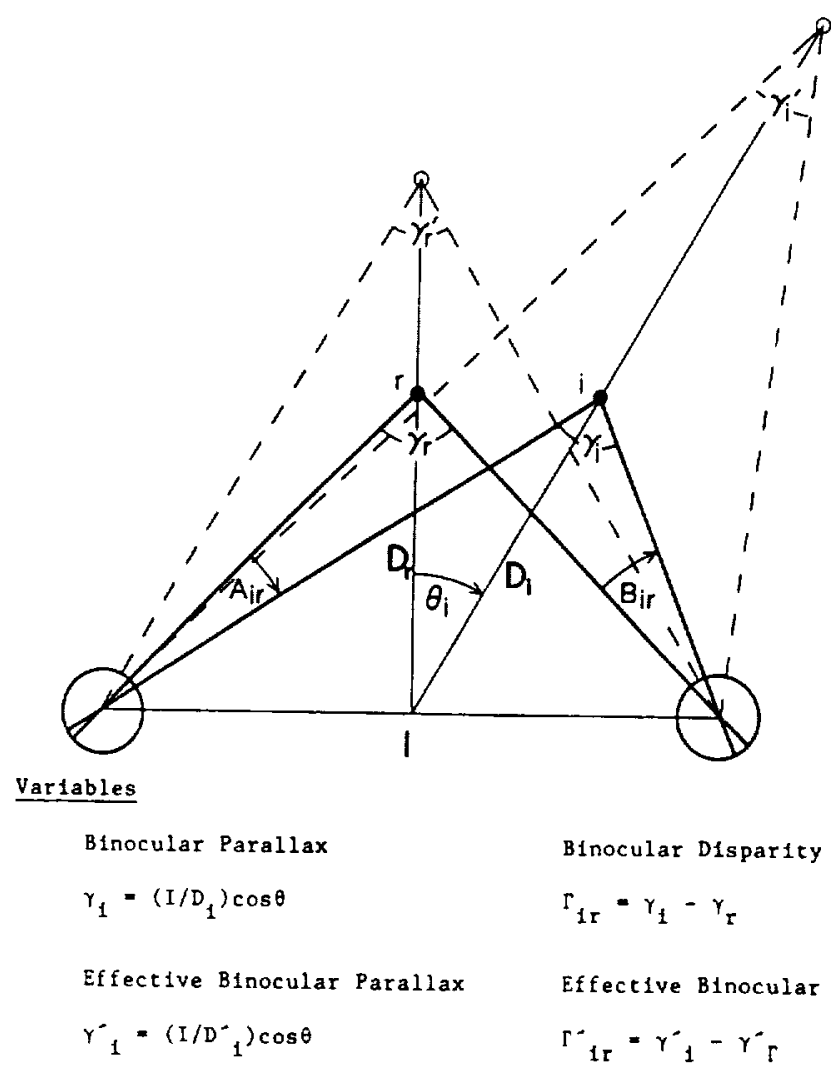

ORIGINAL PAGE IS OF POOR QUALITY

Figure 1.- Variables used in this article. The figure is a top view of the horizontal eye-level plane. The large circles at the bottom represent the two eyes and the solid dots labeled $r$ and $i$ correspond to two stimulus points. The expressions at the bottom of the figure define the four variables. $I$ is interocular distance. $D$ is radial distance to a point. $\theta_{i}$ is horizontal direction of a point relative to straight ahead. $D^{\prime}$ is perceived radial distance.

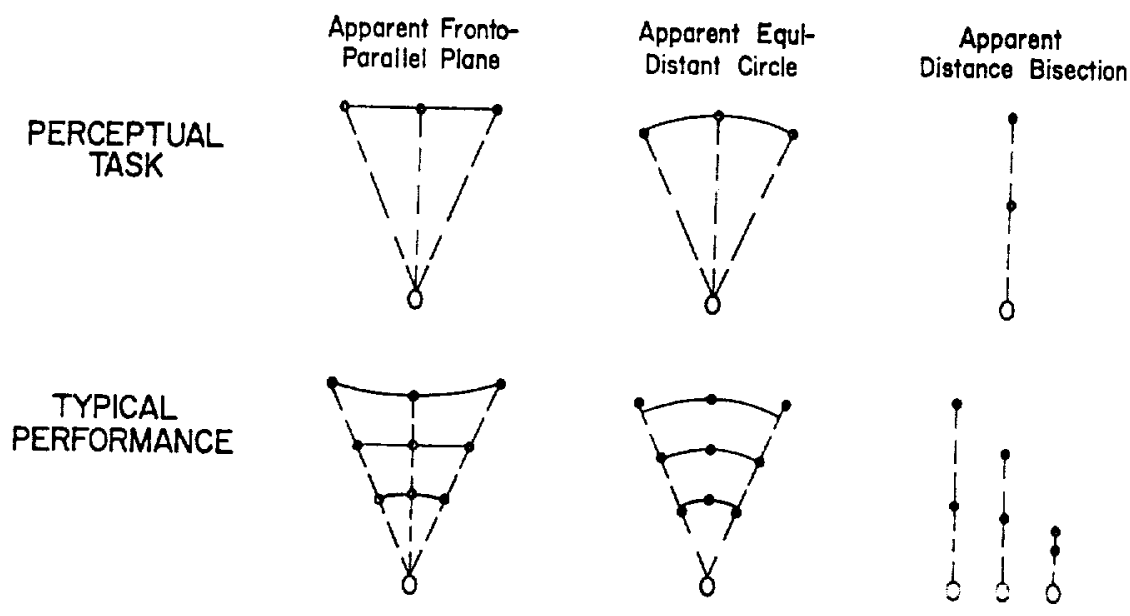

Figure 2.- Illustration of three relative distance tasks (top) and typical performance for observers who show no skewing (bottom). The physical configuration corresponds to the perceptual criterion only at one distance, which is typically between 1 and $4 \mathrm{~m}$. The diagram is not to scale. 


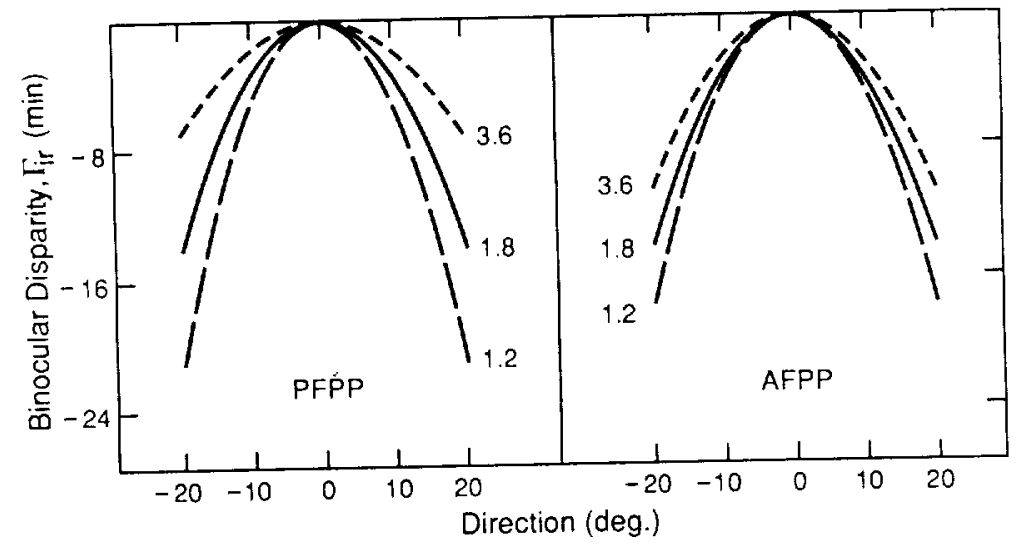

Figure 3.- Binocular disparity as a function of horizontal directions for PFPP and AFPP; the smooth curves describe the results of a typical observer. Each function is shown for three distances of the fixed center point: $1.2,1.8$, and $3.6 \mathrm{~m}$. For this observer the functions correspond at $1.8 \mathrm{~m}$. As distance becomes greater or less than this, the disparities that correspond to the AFPP change less than those corresponding to a PFPP. 

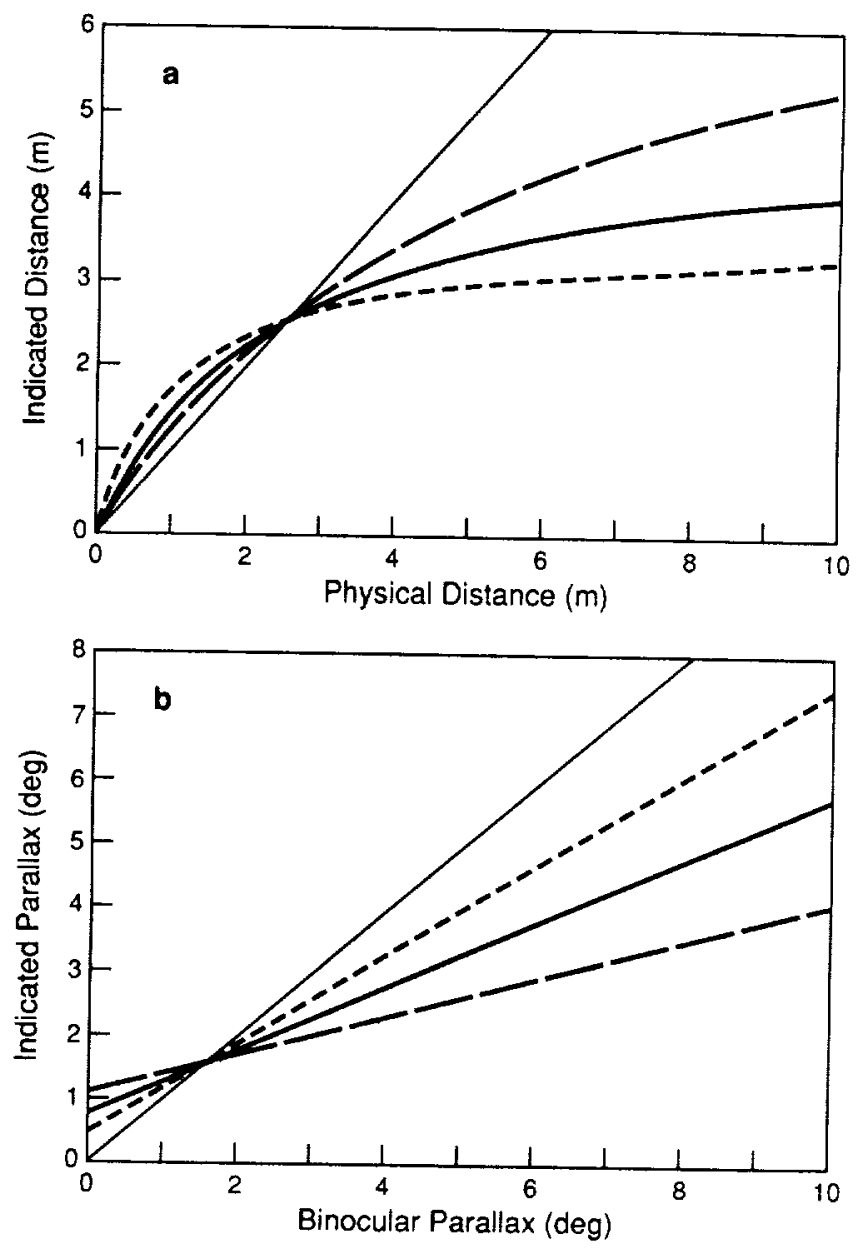

Figure 4.- a) Perceived (or indicated) distance as a function of target distance. Perceived distance inferred from relative distance tasks - ; perceived distance indicated by manual pointing ---; perceived distance indicated by verbal report - - . b) The same three functions expressed as parallaxes. 


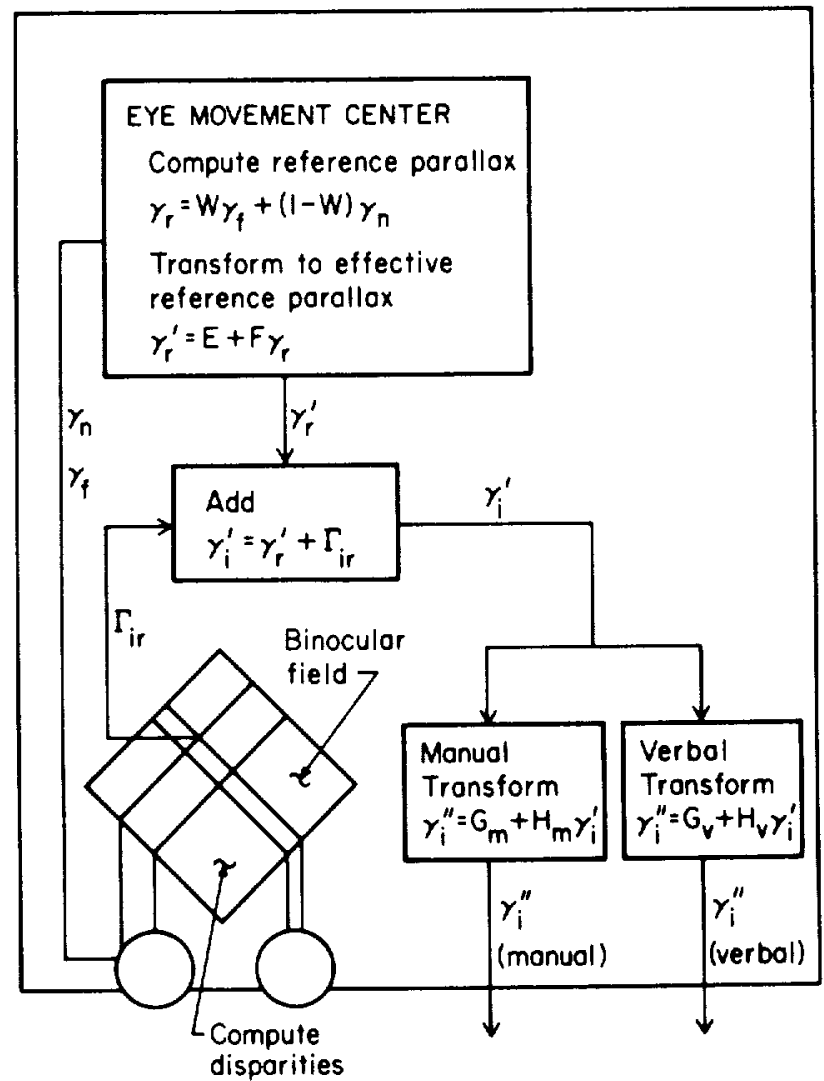

Figure 5.- Diagram summarizing the formal operations of the model in a way that suggests underlying structures and processes (from Foley, 1985). 
